\title{
Correlation between Subliminal Depression and the Quality of Life in Patients Preliminarily Diagnosed with Breast Cancer
}

\author{
Jianjian Chen ${ }^{1}$, Hong Wang2 ${ }^{*}$, Haiyuan Zhang1 ${ }^{*}$, Juan Ding2 \\ ${ }^{1}$ School of Medicine, Yangtze University, Jing Zhou, China \\ ${ }^{2}$ Second Clinical Medicine School of Yangtze University, Breast Department of Jingzhou Central Hospital, Jing Zhou, China \\ Email: 1043969846@qq.com, ^1727923224@qq.com, ^5693293222@qq.com
}

How to cite this paper: Chen, J.J., Wang, H., Zhang, H.Y. and Ding, J. (2019) Correlation between Subliminal Depression and the Quality of Life in Patients Preliminarily Diagnosed with Breast Cancer. Yangtze Medicine, 3, 10-18.

https://doi.org/10.4236/ym.2019.31002

Received: August 28, 2018

Accepted: December 18, 2018

Published: December 21, 2018

Copyright (c) 2019 by authors and Scientific Research Publishing Inc. This work is licensed under the Creative Commons Attribution International License (CC BY 4.0).

http://creativecommons.org/licenses/by/4.0/

\begin{abstract}
Objective: To investigate the subthreshold depression and the quality of life in patients preliminarily diagnosed with breast cancer, and to explore their relationship. Methods: A total of 210 patients preliminarily diagnosed breast cancer were recruited by convenience sampling method to complete the self-made general questionnaire, Center for Epidemiological Studies Depression Scale (CES-D), Hamilton Rating Scale for Depression and Functional Assessment of Cancer Therapy-Breast (FACT-B). Results: The incidence rates of subthreshold depression was $48.6 \%$ in patients preliminarily diagnosed breast cancer, the total score of FACT-B in patients with subthreshold depression and non-subthreshold depression was (73.92 \pm 17.62$)$ and (86.74 $\pm 16.15)$. The total score of CES-D was significantly negatively correlated to the total score and factor scores of FACT-B $(\mathrm{p}<0.01)$. Conclusion: The incidence rate of subthreshold depression in preliminarily diagnosed breast cancer patients is high, and it is closely related to the quality of life, so we should pay attention to the psychological status of preliminarily diagnosed breast cancer patients, to increase the psychological intervention, than improve their quality of life.
\end{abstract}

\section{Keywords}

Patients Preliminarily Diagnosed Breast Cancer, Subthreshold Depression, Quality of Life

\section{Introduction}

Breast cancer is one of the most common malignant tumors in the world, in re- 
cent years, the incidence of breast cancer worldwide has been on the rise, which poses a serious threat to women's physical and mental health [1]. Although the treatment technology of breast cancer is developing and being matured, the survival time of the patients with breast cancer is prolonged [2], but there is still a variety of psychological stress in the treatment of the disease. In the common psychological problems faced by the breast cancer patients, depression is the most popular one [3], but subliminal depression is less attention, patients with newly diagnosed breast cancer should receive more attention due to lack of necessary psychological adjustment [4]. Subthreshold depression (SD) refers to the clinical diagnostic criteria for a partial depression, but not in depression. It is a clinical manifestation between health and depression, a new subtype of depression [4]. Although the clinical symptoms of subliminal depression cannot reach the diagnostic threshold of depression, if they cannot be intervened in time, it will be further developed into depression. There is a literature report that patients with subliminal depression are 5 times more likely to evolve into depression after 1 year than healthy people [5]. There are few studies on subthreshold depression in breast cancer patients. Some studies have reported that middle-aged and young breast cancer patients and long-term survivors of breast cancer have a high incidence of subthreshold depression and have a greater impact on quality of life, which is worthy of attention. The purpose of this study is to investigate the status of subthreshold depression and the quality of life in first diagnosed breast cancer patients, to explore the relationship between them, and to provide a reference for promoting the psychological adjustment and improving the quality of life for the patients with subthreshold depression who are preliminary diagnosed with breast cancer.

\section{Data and Methods}

\subsection{Research Object}

This study included two aspects of the correlation between subliminal depression screening (cross-sectional survey) and subthreshold depression and the quality of life in the first diagnosed breast cancer patients. By using a convenient sampling method, we selected the breast cancer patients in Jingzhou Central Hospital in from January to December in 2017. For the research purposes, we set up inclusion and exclusion criteria, and inclusion criteria were: 1) more than 18 years old; 2) diagnosed as breast cancer for the first time through histopathology; 3) Clear consciousness, no mental disorder; 4) The patients who were included should be with reading and have comprehension skills and be able to complete questionnaires. Exclusion criteria: 1) the patients who had the cognitive abnormality and audiovisual disorder; 2) the patients who disagreed to sign the consent; 3) Patients with previous history of mental illness or organic mental disorder were found to have depression due to psychoactive substances and not addictive substances; 4) Combined with other malignant tumors and those with severe immunodeficiency; 5) Distant metastases. 


\subsection{Methodology}

\subsubsection{Investigative Tools}

1) General situation questionnaire

It was prepared by ourselves, including age, degree of education, marital status, family economic status, occupation, religious belief, medical expense payment, and basic disease.

2) Subthreshold depression initial screening scale flow center depression scale, (CES-D) and hamilton rating scale for depression, (HAMD-17)

The two scales are most commonly used in clinical at home and abroad. CES-D was developed by the National Institute of mental research, Sirodff, in 1977, formerly known as the center for epidemiology, which is used for epidemiological surveys to further examine the target of depressive symptoms. There were 20 items in this scale. Each item was evaluated with one symptom. The total score of 20 symptoms was 0 - 60 points. Among them, 4 items were scored in the opposite direction, and those who scored more than 16 points expressed different degrees of depression. The more the scores were, the higher the degree was. The scale was evaluated according to the frequency of the corresponding situation or feeling in the past week: less than one day was "no or basically no"; the 1 - 2 day was "less"; the 3 - 4 day was "often"; the 5 - 7 day was "almost always". HAMD-17 is used only for CES-D score 16 points for screening of subthreshold depression. It was compiled by Hamilton in 1960. This questionnaire was conducted by two trained assessors for HAMD joint examination. In the way of conversation and observation, two evaluators scored independently after the examination. The severity and treatment effect of the disease were evaluated before and after the treatment. The scores were 7 to normal, and the scores were more than 17 points to depression, between two values, is likely to have depression. At present, there is no unified standard for the diagnosis of subliminal depression at home and abroad. For example, Tan Xi's research method [6], HAMD-17 was used in patients with CES-D score above 16 to screen out patients with depressive episode or pseudo depression. The total scores of CES-D were more than 16, and HAMD-17 were more than 7 and less than 17 as a screening criterion for subliminal depression. However, the criteria for the total scores of CES-D more than or equal to 16 points were criticized by other scholars, because false positives were produced in clinical settings. In this dispute, some scholars have screened severe depression and SD to confirm the scores of CES-D through the Mini International Neuropsychiatric Interview (MINI) questionnaire, and finally determine that CES-D more than 22 can be used as a standard for dividing SD [7]. This study will refer to this standard. The total scores of CES-D were more than 22 points, HAMD-17 were more than 7 points and less than 17 points are the screening criteria for subthreshold depression.

3) Functional assessment of cancer therapy-breast (FACT-B)

This scale is a special scale of the quality of life for breast cancer patients, which is widely used abroad. It was developed by Rush-Presbyterian-St. Luke 
medical center in Chicago, USA [8]. The Functional Assessment of Cancer Therapy-Generic Scale (FACT-G) and the breast cancer specific module are used to measure the quality of life of cancer patients, including physiological, social/family status, emotional status, functional status and additional attention in 5 areas, are made up of 36 items, which $0-4$ points are used to count, the positive and reverse items are converted together, and the higher the score, the better the quality of life and health the patients will get [9].

\subsubsection{Investigation Method}

1) Determination of sample size

The first part of this paper is a cross-sectional study of subthreshold depression screening for newly diagnosed breast cancer patients. The principle of determining the sample size is based on the 5 - 10 times the number of items. There are 20 items of CES-D in this study. Therefore, the range of sample size is $100-200$, but the loss of cases is considered, and the sample amount is increased by $10 \%$, so 210 questionnaires are issued and the same sample size is recovered. The effective rate is $100 \%$.

2) Measuring Method

Under the approval of the Hospital Academic Ethics Committee, a questionnaire survey was conducted by 2 trained researchers to fully explain the purpose and significance of the investigation to the newly diagnosed breast cancer patients who met the inclusion criteria, and to inform the patients that their privacy was kept and own informed consent was obtained. In case of doubt, unbiased guidance was carried out in time. After the completion of the questionnaire, check whether there were blank or invalid items.

3) Statistical Method

SPSS 19.0 software was used for statistical analysis, and measured data were expressed by $\bar{x} \pm \mathrm{s}$, and comparison of two samples was implemented through $t$ test. The enumeration data were expressed by percentages and frequencies and $\chi^{2}$ test was used for comparison of it. The relationship between subliminal depression and the quality of life was analyzed by linear regression analysis.

\section{Results}

\subsection{Demographic Characteristics and Incidence of Subthreshold Depression in Newly Diagnosed Breast Cancer Patients}

All 210 cases of newly diagnosed breast cancer were women, aged 19 - 65 years, with an average of $(47.1 \pm 8.28)$ years. By investigating the total score of CSE-D, there were 93 cases of no depression. 102 cases among 210 patients suffered from subthreshold depression, and 15 cases of depressive symptoms, accounting for $7 \%$. The age, marital status, family monthly income, on-the-job status, educational level, and subthreshold depression rate of basic diseases were statistically significant $(\mathrm{p}<0.01)$, and the rest of the cases were shown in Table 1. 
Table 1. Social demographic characteristics and incidence of subthreshold depression in newly diagnosed breast cancer patients with subthreshold depression (cases, percentage (\%)).

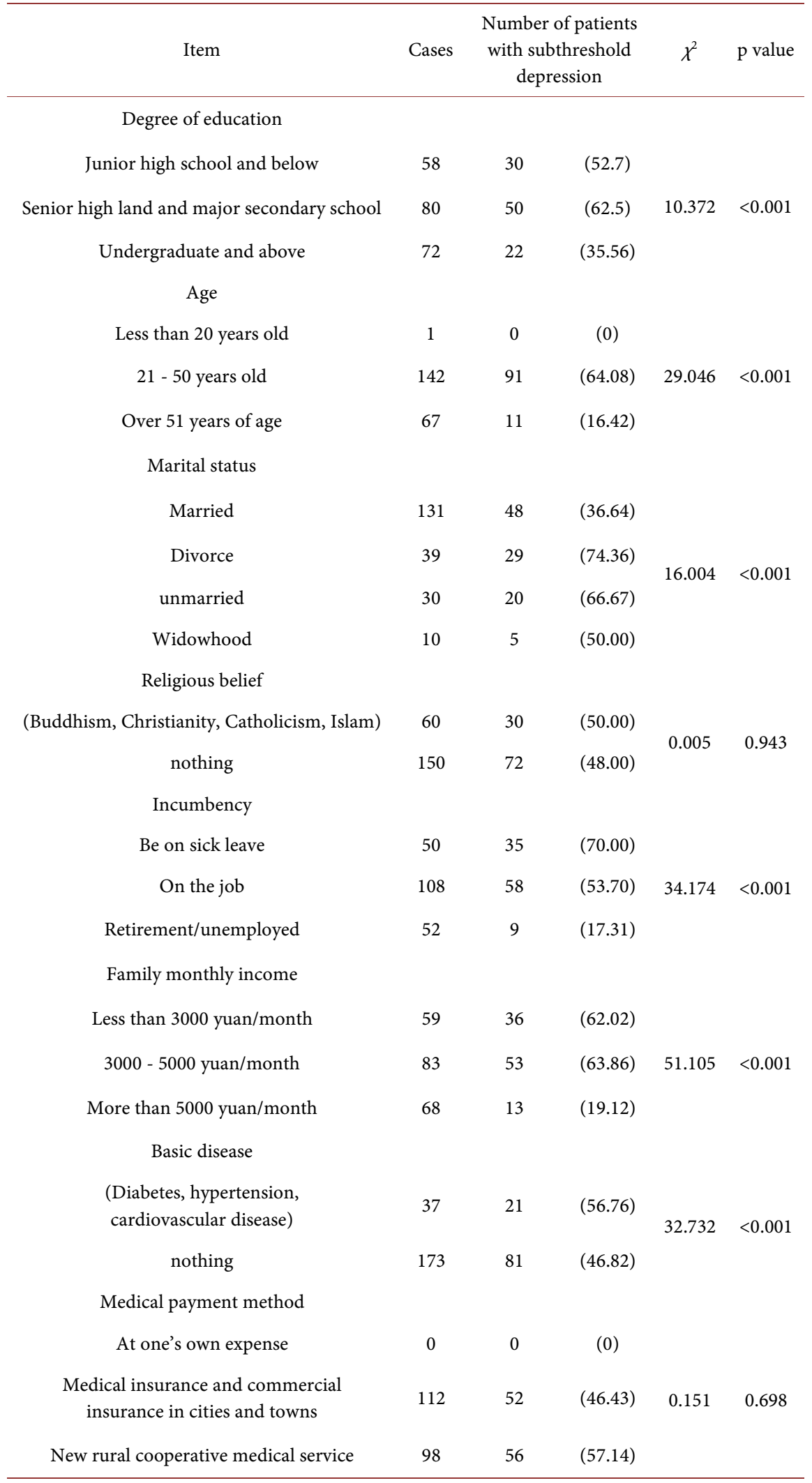




\subsection{The Score of the Quality of Life in Patients with Newly Diagnosed Breast Cancer with Subthreshold Depression and without Depression}

In this survey, the total scores of FACT-B in patients with subthreshold depression in first diagnosed breast cancer were $(73.92 \pm 17.62)$, and the total scores of FACT-B in non-depressive group patients were $(86.74 \pm 16.15)$, and the scores of each dimension were shown in Table 2. The scores of all dimensions of the FACT-B scale in the subthreshold depression group were lower than those in the non-depression group, $\mathrm{p}<0.01$, the difference was statistically significant.

\subsection{Correlation between Subliminal Depression and the Quality of Life in Newly Diagnosed Breast Cancer Patients}

The total scores of the quality of life and the scores of all dimensions in 195 respondents were dependent variables, and linear regression analysis with CES-D scores as independent variable. CES-D was negatively correlated with the dimensions of the FACT-B scale. Subliminal depression had a great influence on all fields of the quality of life in the patients primarily diagnosed breast cancer, and the greatest impact on the relationship dimension of physiological status and functional status. The study results were shown in Table 3.

Table 2. Comparison of the quality of life among the two groups of newly diagnosed breast cancer patients. (Fraction, $\bar{x} \pm \mathrm{s}$ ).

\begin{tabular}{|c|c|c|c|c|c|c|c|}
\hline Group & Cases & $\begin{array}{c}\text { Physiological } \\
\text { status }\end{array}$ & $\begin{array}{c}\text { Social/family } \\
\text { status }\end{array}$ & $\begin{array}{c}\text { Emotional } \\
\text { status }\end{array}$ & $\begin{array}{c}\text { Functional } \\
\text { status }\end{array}$ & $\begin{array}{l}\text { Additional } \\
\text { attention }\end{array}$ & Total score \\
\hline $\begin{array}{c}\text { Subthreshold } \\
\text { depression }\end{array}$ & 102 & $15.03 \pm 5.89$ & $15.48 \pm 4.83$ & $13.41 \pm 5.07$ & $9.41 \pm 4.77$ & $21.11 \pm 5.07$ & $73.92 \pm 17.62$ \\
\hline $\begin{array}{c}\text { Non } \\
\text { depression }\end{array}$ & 93 & $19.34 \pm 4.41$ & $17.67 \pm 4.37$ & $14.73 \pm 4.54$ & $12.41 \pm 5.07$ & $24.01 \pm 4.97$ & $86.74 \pm 16.15$ \\
\hline $\mathrm{t}$ value & & -6.98 & -3.78 & -2.98 & -4.45 & -3.98 & -6.73 \\
\hline $\mathrm{p}$ value & & $<0.001$ & $<0.001$ & 0.003 & $<0.001$ & $<0.001$ & $<0.001$ \\
\hline
\end{tabular}

Table 3. Linear regression analysis of the dimensions of CES-D and quality of life.

\begin{tabular}{cccccccc}
\hline Dimensions & $\mathrm{B}$ & $\beta$ & $\mathrm{SE}$ & $t$ & $\mathrm{p}$ & $\mathrm{R}^{2} \begin{array}{c}\text { Correction } \\
\text { of } \mathrm{R}^{2} \text { value }\end{array}$ \\
\hline Physiological status & -0.305 & -0.454 & 0.065 & -5.025 & $<0.001$ & 0.128 & 0.125 \\
Social/family status & -0.153 & -0.347 & 0.047 & -4.332 & $<0.001$ & 0.727 & 0.073 \\
Emotional status & -0.104 & -0.180 & 0.056 & -3.498 & 0.041 & 0.063 & 0.046 \\
Functional status & -0.184 & -0.312 & 0.051 & -4.687 & $<0.001$ & 0.121 & 0.096 \\
Additional attention & -0.173 & -0.320 & 0.056 & -3.873 & $<0.001$ & 0.766 & 0.067 \\
Total score & -0.988 & -0.678 & 0.077 & -12.831 & $<0.001$ & 0.460 & 0.458 \\
\hline
\end{tabular}

Note: CES-D is the flow center depression scale. 


\section{Discussion}

\subsection{The Occurrence of Subthreshold Depression in First Diagnosed Breast Cancer}

This study showed that $48.6 \%$ of patients preliminarily diagnosed with breast cancer had subliminal depression, of which the incidence of subthreshold depression in first diagnosed breast cancer patients aged 21 - 50 was $64.08 \%$, which was higher than that of Lv Liming [10] [11]. The reasons may be as follows: This study is only based on the first diagnosis of breast cancer patients. When the patient is diagnosed as breast cancer, it has just started to be in a stressful state, and it needs a certain process for the acceptance of the reality. There are puzzling, nervous and worried feelings on the treatment plan and effect, and in the treatment of late disease, there will be a relatively sufficient time for psychological adjustment. This study found that low income, divorced/unmarried/widowed, sick/retired/unemployed, young and middle-aged first diagnosed breast cancer patients were prone to subliminal depression, which was consistent with the results of Lv Liming and other studies [10] [11]. The incidence of subthreshold depression was lowest among undergraduates and above, indicating that the higher education level of newly diagnosed patients was, the better knowledge of disease the people could get. This can reduce anxiety and panic about their own diseases. It also suggests that medical staff should use a language that is easy to understand and to be more patient, so that patients can acquire and understand more related knowledge of diseases and reduce the fear of diseases. In basic diseases (diabetes, hypertension, cardiovascular disease), the incidence of subliminal depression in first diagnosed breast cancer patients with basic diseases is higher than those without basic diseases. The patient's own basic disease and the diagnosis of breast cancer, which further aggravates the patient and family burden, then it has an impact on the patient's psychology. Although the incidence of subliminal depression in first diagnosed breast cancer patients is high, most patients and families have not enough attention to mental illness especially subliminal depression, and the symptoms of subliminal depression are easily confusing with other diseases, causing such patients not to receive professional treatment for the changes in physical symptoms and let it develop at any time. Therefore, early diagnosis of breast cancer patients also require a certain psychological adjustment process, the medical staff should pay more attention to the psychological problems of the people with high incidence, to find and take effective measures in time to avoid the further development of their psychological diseases.

\subsection{Relationship between Subliminal Depression and the Quality of Life in the First Diagnosis of Breast Cancer}

The results showed that the total scores of the life quality and the scores in all areas were significantly lower than those in the non-depression group. The depression score of preliminarily diagnosed breast cancer patients was negatively 
correlated with the quality of life, that is, the higher the score of depression, the lower the quality of life. Among them, subliminal depression has a significant effect on physiological and functional status. On the one hand, depression tends to increase the sensitivity of the patients to physical discomfort, on the other hand, depression can also cause and aggravate physical symptoms, in line with the results of the research [12]. In addition, subthreshold depression is often in the state of self-degrading, coupled with continuous fatigue to reduce the emotional experience, the willingness and initiative to participate in social activities and the decline in the quality of life [13]. Studies also have shown that cancer related depression plays an important role in the development and prognosis of cancer, it can affect the effect and compliance of cancer treatment, cancer recurrence, metastasis and deterioration also will be promoted, thus causing the quality of life of the patients reduced [14].

\section{Limitation}

From the perspective of first diagnosis of breast cancer, this study can provide early screening for patients with subthreshold depression of breast cancer and provide theoretical basis for the early detection of psychological diseases. However, the investigation is limited to a level-three class-A hospital in Jing Zhou, it also needs multi-regional and comprehensive survey data to support. So far, there are no specific criteria for assessing subliminal depression in breast cancer patients, and further in-depth study is needed to develop a more suitable standard for subliminal depression in Chinese breast cancer patients.

\section{Conflicts of Interest}

The authors declare no conflicts of interest regarding the publication of this paper.

\section{References}

[1] Yuan, H.R., Liang, Y., Cheng, N., Xie, X.L. and Zhao, L.Y. (2018) 60 Cases of Breast cancer Postoperative Rehabilitation Training and Nursing Practice. Journal of Nursing, 25, 67-69.

[2] Bai, Z.J. and Li, X.L. (2012) Influence of Group Psychotherapy on Anxiety and Depression of Breast Cancer Patients. Chinese Journal of Health Psychology, 20, 1367-1369.

[3] Huang, M.Q., Wu, T., Zhang, Q.Y., Wang, Y., Pang, W. and Yan, L. (2018) The Mediating Effect of Symptom Management Self-Efficacy on Anxiety and Depression and Quality of Life in Patients with Breast Cancer during Chemotherapy. Journal of Nursing, 25, 65-69.

[4] Liu, Y., Tan, X., Tian, Y.Q., Du, Y. and Kong, J.H. (2014) Epidemiology and Related Research of Subliminal Depression. Chinese Journal of Medicine, 29, 1511-1513.

[5] Liu, Y., Tan, X., Zhang, J., Qiao, Y., Chen, L.X., Yu, L.L., et al. (2015) Current Status and Prospect of Subliminal Depression Recognition. World Journal of Traditional Chinese Medicine, 10, 798-800. 
[6] Tan, X., Yang, Q.L., Du, Y., Wu, Z.Y. and Kong, J.H. (2012) Group Psychotherapy Intervention Program for Patients with Subthreshold Depression. Journal of Traditional Chinese Medicine, 53, 2087-2089.

[7] Gong, X. and Cai, T.S. (2009) Overview of Research on Subliminal Depression. Medicine and Philosophy (Humanities and Social Medicine Edition), 30, 47-48.

[8] Sun, L.Y. (2016) Analysis of the Influencing Factors of Disability Acceptance after Breast Cancer Surgery and the Effect of Group Psychological Counseling Intervention. Tianjin Medical University, Tianjin.

[9] Li, P., Chen, L., Wei, Y.J., Li, W., Liao, H.F., Gao, H., et al. (2017) Research on the Application of Quality of Social Relations in the Field of Nursing Care. Nursing Research, 31, 3095-3096.

[10] Lv, L., Zhang, X.H., Wang, X.X. and Zhang, J.L. (2017) Subthreshold Depression and Social Participation in Young and Middle-Aged Patients after Breast Cancer Surgery. Journal of Nursing Science, 32, 84-87.

[11] Lv, L., Gao, H., Cheng, H.Y., et al. (2017) A Study on the Correlation of Subthreshold Depression and Quality of Life of Long-Term Survivors after Breast Cancer Surgery. Chinese Journal of Practical Nursing, 33, 259-264.

[12] Lyness, J.M., Heo, M., Datto, C.J., et al. (2006) Outcomes of Minor and Subsyndromal Depression among Elderly Patients in Primary Care Settings. Annals of Internal Medicine, 144, 496-504.

https://doi.org/10.7326/0003-4819-144-7-200604040-00008

[13] Carlson, L.E., Beattie, T.L., Giese-Davis, J., et al. (2015) Mindfulness-Based Cancer Recovery and Supportive-Expressive Therapy Maintain Telomere Length Relative to Controls in Distressed Breast Cancer Survivors. Cancer, 121, 476-484. https://doi.org/10.1002/cncr.29063

[14] Zhu, L., Xu, S., An, Y.Y. and Chen, X.L. (2018) The Effect of Posttraumatic Stress Disorder on quality of Life in Patients with Breast Cancer: Mediating Effect of Depression and Caregiver Stress. China Journal of Health Psychology, 26, 225-229. 